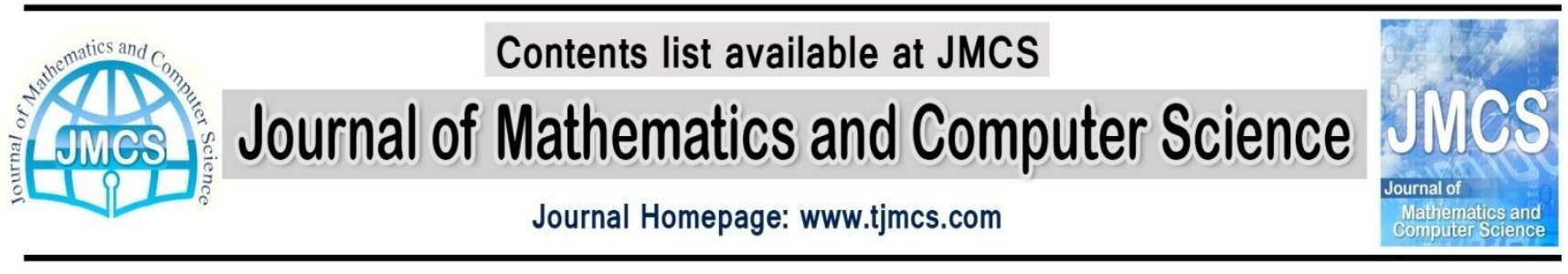

\title{
Comparison between Topological Properties of HyperX and Generalized Hypercube for Interconnection Networks
}

\author{
Sadoon Azizi ${ }^{1 *}$, Naser Hashemi ${ }^{1}$, Mohammad Amiri Zarandi ${ }^{2}$ \\ ${ }^{1}$ Department of Mathematics and Computer Science, Amirkabir University of Technology, \\ Tehran, Iran \\ ${ }^{2}$ Department of Mathematics and Computer Science, Shahid Bahonar University, Kerman, Iran \\ s.azizi@aut.ac.ir \\ nhashemi@aut.ac.ir \\ mohammadamiri@uk.ac.ir
}

Article history:

Received August 2013

Accepted November 2013

Available online November 2013

\section{Abstract}

In order to design an interconnection network, it is essential to have a comprehensive understanding about properties and limitations of the network. These properties and limitations are characterized by the topology of the network. Since a topology sets constraints and costs, it plays a critical role in all interconnection networks. Different topologies have been proposed for interconnection networks in literature. The Generalized Hypercube is one of the oldest topologies that can be mentioned. Recently a group of researchers at HP Lab have introduced a new topology for these networks, called HyperX. Despite of many similarities between these two topologies, there are significant differences between their performances and costs. It seems that this important issue has been neglected in contexts of interconnection networks. In this paper, we compare HyperX and Generalized Hypercube topologies under some key topological measures. We show that HyperX is somehow better than Generalized Hypercube in the sense of topological properties.

Keywords: Topological properties, HyperX, Generalized Hypercube, Interconnection Networks, Performance. 


\section{Introduction}

Network topology determines the structure of the interconnection network. In other words, topology shows the static order of terminals, switches (or routers) and links. Topology is one of the important aspects of any interconnection network; because it sets many limitations of the network includingdegree, diameter, average distance and the number of links [1].

In the literature of interconnection networks, different topologies have been presented by researchers. For instance, we can mention Mesh, Torus, Hyper cube and Generalized Hypercube topologies [1]. During the past few years, increasing integrated-circuit pin bandwidth has increased the radix of interconnection networks and their switches. Exploitation of the high-radix switches has led to higher performance and lower cost [2]. This follows emergence of new topologies for the interconnection networks. First, in 2007, Kim et al. [3] introduced the new high-radix topology, named Flattened Butterfly. Two years later, Ahnet al. [4] at HP Lab proposed another new highradix topology called HyperX. This topology can be considered as a general framework for other well-known topologies including Hypercube and Flattened Butterfly [4]. It is necessary to mention that HyperX is also a general framework forGeneralized Hypercube, as well. But, this issue has not been pointed out in the reference [4] and we will briefly show it in Section 2 of this paper.

HyperX and Generalized Hypercube topological structure are slightly different, but this slight differencehas made a huge difference on performance and cost of them. So far in the literature of the interconnection networks, there is no comparison between these two topologies. Therefore, in this paper we are going to focus on this issue. With this study, we are able to achieve a comprehensive understanding about the performance and cost of each topology and their comparison.

Therest of the paper is organized as follows. The description of two topologies structure is presentedinSection 2. In Section 3, the most important performance and cost measures are introduced and we extract them for the studied topologies.In Section 4these two topologies are compared together. Finally, Section 5 concludes the paper and proposes suggestions for future work.

\section{Definitions and preliminaries}

\subsection{Describing Generalized Hypercube topology and its model}

The Generalized Hypercube topology was introduced by Bhuyan and Agrawal in 1984 [5]. They also extracted some of its topological properties such as average distance, diameter, and number of links. Generalized Hypercubeis a kind of direct interconnection networks such that one terminal is exactly connected to each switch.This topology, in fact, is a generalization of Hypercube. That means, we can put more than two switches in each dimension with complete connections. If we assume that $N$ is the number of nodes (switches), $n$ number of dimensions and $m_{i}$ the number of nodes in dimension $i, 1 \leq i \leq n$, then Generalized Hyper cube can be shown with the symbol $\mathrm{GHC}_{\mathrm{m}_{1}, \mathrm{~m}_{2}, \ldots, \mathrm{m}_{\mathrm{n}}}$ and is defined as follows.

Definition 1[5]: Each node like $A, 0 \leq A \leq N-1$, can be identified as a vector of $n$ coordinates $\left(a_{n}, a_{n-1}, \ldots, a_{1}\right)$ such that $a_{i}, 0 \leq a_{i} \leq\left(m_{i}-1\right)$ indicating the location of nodes in dimension $i$. In Generalized Hypercube topology, each node is connected to all nodes that differ only in one dimension.In other words, the node $\left(a_{n}, a_{n-1}, \ldots, a_{i+1}, a_{i}, a_{i-1}, \ldots, a_{1}\right)$ in dimension iis 
connected toall nodeswith vector coordinates $\left(a_{n}, a_{n-1}, \ldots, a_{i+1}, b_{i}, a_{i-1}, \ldots, a_{1}\right)$ where $0 \leq b_{i} \leq$ $\left(m_{i}-1\right), b_{i} \neq a_{i}$.

Figure 1 demonstrates two-dimensional Generalized Hypercube. The number of nodes in the first dimension equals 2 and in the second dimension are 4.

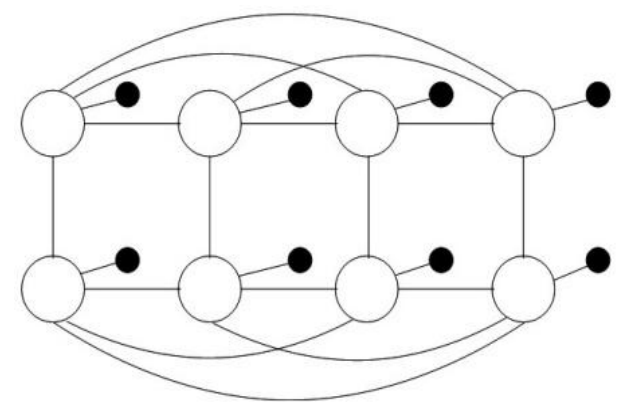

Figure 1: A 2-dimensionalGeneralized Hypercube topology. White circles are nodes (switches) and black circles are terminals.

\subsection{Describing HyperX topology and its model}

Several years after introducing Generalized Hypercube topology, HyperX was introduced by a group of researchers at HP Lab in 2009 [4]. HyperX, also, is a direct network of switches in whichthe constant number of $T$ terminals is exactly connected to each switch. Each terminal can be a computational node, cluster of computational nodes, I/Onode, or any other interconnection devices. Nodes (switches), such as Generalized Hypercube topology, are organized in several dimensions with complete connections in each dimension. Obviously, if $T>1$ the number of switches will be less than the number of terminals. It is assumed that $P$ is the number of switches in the network, and $r_{i}$ is the number of switches in dimension $i$. Therefore, $\mathrm{HX}_{\mathrm{r}_{1}, \mathrm{r}_{2}, \ldots, \mathrm{r}_{\mathrm{n}}}$ represents the $\mathrm{n}$-DHyperX topology. Here, it is important to note that Azizi et al. [9] have recently investigated the topological properties of HyperX network in a comprehensive study.

Definition 2[9]: In HyperX each node such $A, 0 \leq A \leq P-1$ can be represented by n-tuple coordinate vector $\left(a_{n}, a_{n-1}, \ldots, a_{1}\right)$ in which $a_{i}, 0 \leq a_{i} \leq\left(r_{i}-1\right)$ denotes the position of the node in dimension i. In HyperX network each node is connected to all nodes that differ only in one dimension. In other words, the node $\left(a_{n}, a_{n-1}, \ldots, a_{i+1}, a_{i}, a_{i-1}, \ldots, a_{1}\right)$ in dimension $\mathrm{i}$ is connected toall nodes with coordinate vector $\left(a_{n}, a_{n-1}, \ldots, a_{i+1}, b_{i}, a_{i-1}, \ldots, a_{1}\right)$ in which $0 \leq b_{i} \leq$ $\left(r_{i}-1\right), b_{i} \neq a_{i}$.

Figure 2 depicts 2-DHyperXtopology. In this figure, 16 terminals physically contacted with each other through a HyperX topology. Here, the same as Figure 1, the number of nodes in the first dimension is 2 and the second dimension equal 4. 


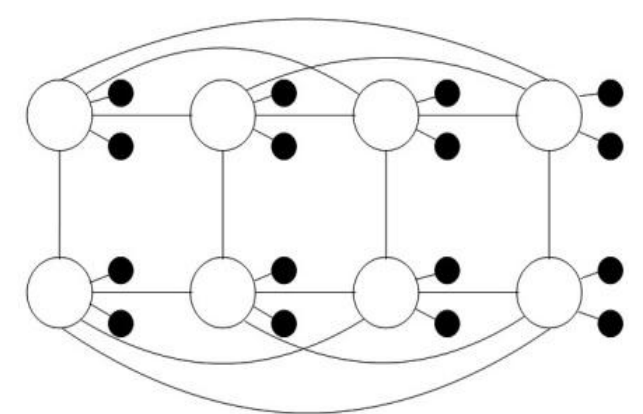

Figure2. HyperX topology. White circles are switches and the black ones are terminals $(\mathrm{T}=2)$.

As can be inferred from two topologies structure, there is a very strong resemblance between them and the only difference is the number of terminals connected to each node. For Generalized Hypercube $T=1$ and this value can be any constant number for HyperX, that means $T \geq 1$. It can be clearly realized that Generalized Hypercube is a special case of HyperX. In other words, HyperX is considered asa framework for Generalized Hypercube.

\section{Performance and cost measures}

In $[6,10]$ several important measures have been listed in order to evaluation of interconnection networks including diameter, average distance, number of switches, number of links and network degree.The diameter and the average distance can be considered asperformancemetricsand the number of switches, the number of links and the degree of a networkare cost metrics. In this section wefirst begin by reviewing their definitions and thenwe extractthemfor our studied topologies.It should be mentioned that some of them have been calculated before [4-5, 9].

\subsection{Evaluation measures}

Definition 3[6] (Diameter of the network, D): The longest distance between each pair ofnodes in thenetwork stands for the diameter of that network.

Definition 4 [5] (Average distance, $\overline{\mathrm{D}}$ ): The average distance between all pairs of nodes in a networkis the average distance of thatnetwork andis given by

$$
\overline{\mathrm{D}}=\frac{\sum_{\mathrm{i}=1}^{\mathrm{n}} \mathrm{i} \times \mathrm{N}_{\mathrm{i}}}{\mathrm{N}-1}
$$

Where $\mathrm{N}$ is the number of network terminals and $\mathrm{N}_{\mathrm{i}}$ is defined by the set of nodes located in distance $\mathrm{i}$ from the central node $\mathrm{O}$.

Definition 5[6] (Network degree, $\Delta$ ): The node with the biggest degree in the network determines the degree of that network.

Remark 1: In this paper, the inner links (links between nodes and terminals are also considered for computing the number of links in the network to makea fair comparison between the two topologies. 
Definition 6[6] (Number of switches, S):The number of switchesneeded to build a network of size N.

Definition 7[6] (Number of links, E): The links needed to build a network of sizeN and its formula is obtained by

$$
\mathrm{E}=\frac{\mathrm{S} \times \Delta}{2}
$$

As noted above, Sand $\Delta$ are the number of switchesand the networks degree, respectively.

\subsection{Assessment of measures for studied topologies}

\subsubsection{Generalized Hypercube}

Bhuyan and Agrawal [5] have extracted some parameters for Generalized Hyper cube. Here, we restate them by considering remark 1 .

Network diameter,

$$
\mathrm{D}_{\mathrm{GHC}}=\mathrm{n}
$$

Average distance,for the case that $\left(m_{i}=m, 1 \leq m_{i} \leq n\right)$

$$
\overline{\mathrm{D}}_{\mathrm{GHC}}=\mathrm{n} \cdot(\mathrm{m}-1) \cdot \mathrm{m}^{\mathrm{n}-1} /(\mathrm{N}-1)
$$

Network degree,

$$
\Delta_{\mathrm{GHC}}=1+\sum_{\mathrm{i}=1}^{\mathrm{n}}\left(\mathrm{m}_{\mathrm{i}}-1\right)
$$

Number of switches,

$$
\mathrm{S}_{\mathrm{GHC}}=\mathrm{N}
$$

Number of links,

$$
\mathrm{E}_{\mathrm{GHC}}=\mathrm{N}\left(1+\left(\sum_{\mathrm{i}=1}^{\mathrm{n}}\left(\mathrm{m}_{\mathrm{i}}-1\right)\right) / 2\right)
$$

\subsubsection{HyperX}


Ahn and his collaborators [4] in their paper focused ondescription of HyperX topology and its comparison with folded Close topology [7]. Finally, they proposed a routing algorithm called DAL that attempt to balance the traffic load throughout the network. However, the topological properties of their topology have been ignored. In [9] this issue have been done by Azizi et al. Here, we explain some of them for our task in this paper.

The diameter of HyperX network, like Generalized Hypercube, is equal to the number of network dimensions. That is true becauseone of dimension differencecan be compensated with each step. And since a pair of nodes in HyperX in the worst case are different in all dimensions, so the network diameter is the same as the number of dimensions.

$$
\mathrm{D}_{\mathrm{HX}}=\mathrm{n}
$$

The following is the average distance of HyperX. The proof is given in the appendix of this paper.Here to express the average distance with aformula,its regular mode, i.e. $\left(1 \leq r_{i} \leq\right.$ $n, r_{i}=r$ ), have been considered.

$$
\overline{\mathrm{D}}_{\mathrm{HX}}=\mathrm{n} \cdot \frac{\mathrm{r}-1}{\mathrm{r}} \cdot \frac{\mathrm{N}}{\mathrm{N}-1}
$$

Since HyperX is a regular network, its degree is equal to the degree of each of node. Each node is connected to all nodes that have difference only in one dimension with it. Besides, each node is connected exactly to $T$ terminals. Then, the topology degree of HyperX is equal to

$$
\Delta_{\mathrm{HX}}=\mathrm{T}+\sum_{\mathrm{i}=1}^{\mathrm{n}}\left(\mathrm{r}_{\mathrm{i}}-1\right)
$$

In HyperX, unlike Generalized Hypercube, the number of switches is less than or equalto the number of terminals (network size).Because each switch hasTterminals. So we have

$$
\mathrm{S}_{\mathrm{HX}}=\mathrm{N} / \mathrm{T}
$$

The number of links in HyperX can be calculated with replacement of expressions (10) and (11) in equation (2). Thus, we have

$$
\mathrm{E}_{\mathrm{HX}}=\mathrm{S}_{\mathrm{HX}}\left(\mathrm{T}+\left(\sum_{\mathrm{i}=1}^{\mathrm{n}}\left(\mathrm{r}_{\mathrm{i}}-1\right)\right) / 2\right)
$$

4. Comparison of performance and cost of HyperX and Generalized Hyper cube

In this section, we compare the topological structure of HyperX and Generalized Hyper cube under measures described in subsection 3.1.

4.1. Network diameter 
As the equation (3) and (8) denotes, the diameter of two topologies is the same i.e., their diameter equal to the number of network dimensions, $n$.

\subsection{Average distance}

To compare the average distance of two topologies, the size of network is assumed between 16 and 4096. The motivation for this choice is that we want to measure the average distance between two networks for small-scale and large-scale ones.The simulation resultis given in Figure 3. As can be inferred from the result, the average distance of HyperX is less than Generalized Hypercube in any size.

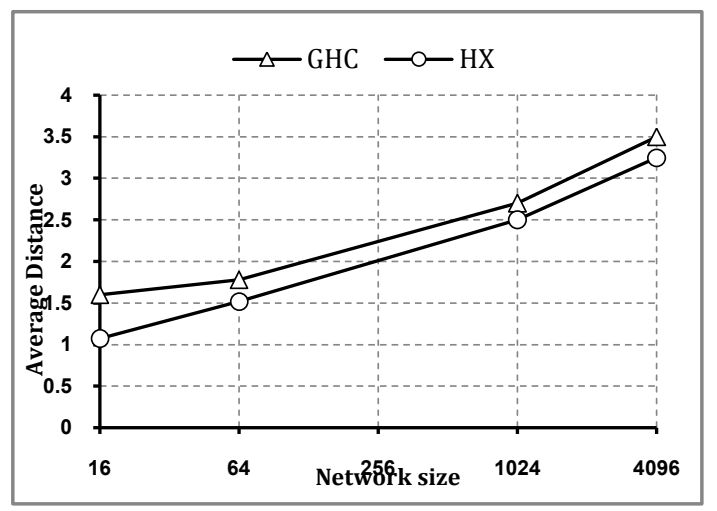

Figure3. Comparison of HyperX and Generalized Hypercubeaverage distance

\subsection{Network degree}

To compare the degree of HyperX and Generalized Hypercube, we assume the number of dimensions the same, also, for HyperX $T=4$. As Figure 4 demonstrates, HyperX degree is less than Generalized Hypercube.

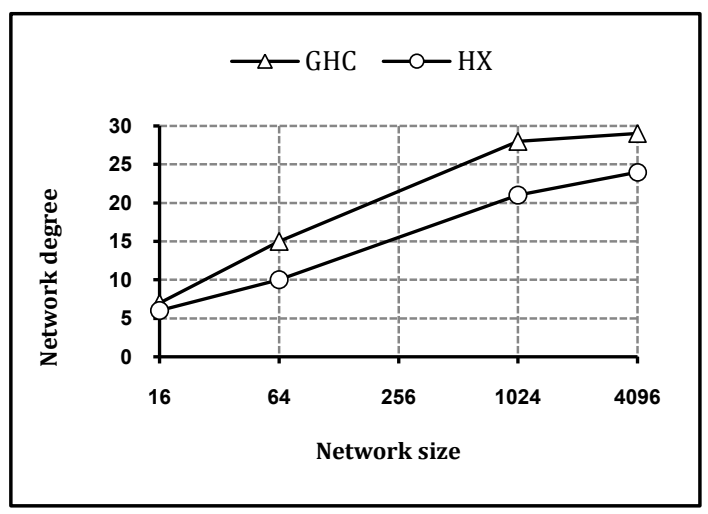


Figure 4. Comparison of HyperX and Generalized Hypercube network degree

\subsection{Normalized average distance}

Topologies with low average distance usually have high network degree. Thus, it is important to have a measure that take into account both of them as a measure. Normalized average distance is such criterion that has been introduced by Lee and Ganz [11]. It is defined as the product of average distance and network degree. Actually, this measure is a trade-off between the average distance and the degree of a network. Therefore, we compare the HyperX and Generalized Hypercube under it. As figure 5 indicates, HyperX is good in this measure, too. The network with low average distance usually has better performance. Of course, the routing algorithm play a critical role to achieve good performance and QoS. For example, one can use heuristic strategies to do this [12].

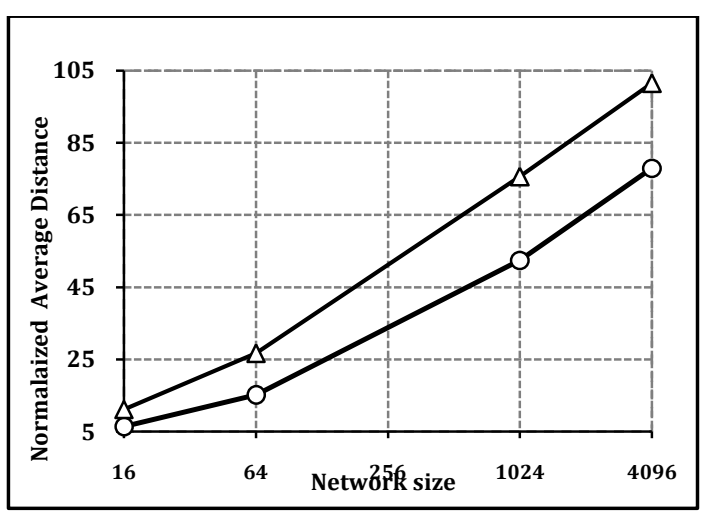

Figure 5. Comparison of HyperX and Generalized Hypercube network degree

\subsection{Number of switches}

By comparing related expressions for the number of switches in Generalized Hyper cube and HyperX topology, it can be found that the difference between them is the amount of T. It is clear that, on the one hand, the number of switches in the network decrease as the amount of T increases, andon the other hand, higher $\mathrm{T}$ increase the degree of switches. Therefore, in order to make a fair comparison between thenumber ofswitches in the two topologies, we consider theswitch degreethe same asFigure 4.As figure 6shows for building a fixedsize network, HyperX topology will require fewer switches compared with the Generalized Hypercubetopology. It is important to note that the degree and the number of switches in HyperX topology are less than Generalized Hypercube, simultaneously. 


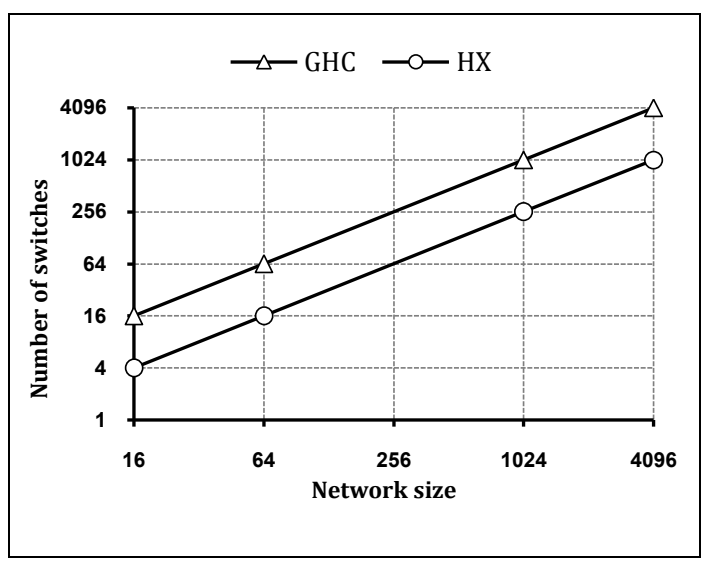

Figure 6. Comparison of the number of HyperX and Generalized Hypercube switches

\subsection{Number of links}

In this subsection, we compare the number of links used by two topologies. We assumed $\mathrm{T}=4$ for HyperX topology. The result, Figure 7, shows that HyperX needs fewer links to compare with Generalized Hypercube.

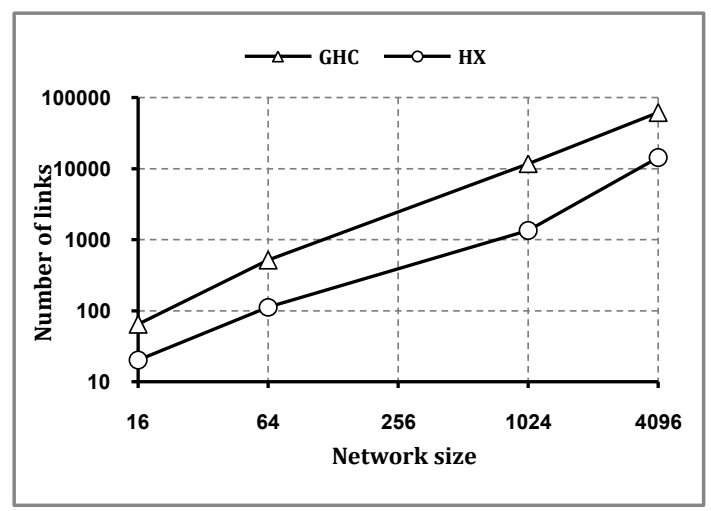

Figure 7.Comparison of the number of HyperX and Generalized Hypercubelinks

\section{Conclusions and Future Works}

Due to progress of technology and its effects on the computer architecture, evaluation of this field has been become an important issue. The technology of signaling and also total bandwidth of chip pins has a substantial effect on the performance of interconnection networks. This development has introduced a new type of networks that are called high radix routers.HyperXis an obvious example of this architecture. Our main contribution in this paper is extracting some aspects of off-chip HyperX topology using mathematical expressions. Extracting these kinds of expressions in order to describe topological properties of HyperX can have several benefits. For example, determining the structural properties of networks can be very helpful in designing graph algorithms in order to determine communication patterns between nodes, designing and implementing the routing algorithms, 
investigating resource replacement and their allocation, implementing VLSI layout, examining network vulnerability against attacks and failures and finally analytical modeling and performance evaluation of the network.

With advent of new topologiestothe network arenathere is a need to compare its structure with other topologies presented in the literature. One of literature topologies is interconnection network of Generalized Hypercube that has a remarkable similarity with HyperX topology in appearance but their structural parameters are quite different.Therefore, the focus on the second part of this paper is the comparison of HyperX and Generalized Hypercubeunder several important performance and cost measures. The results show that HyperX has better performance and cost than Generalized Hypercubein terms of structural properties.Another future work can be the evaluation ofHyperX and Generalized Hypercube network under important criteria in field of networker chips (NoCs). Also, we can compare these two topologies with considering the functional measures; i.e., take into account the traffic engineering within the network.

\section{Appendix}

The proof of average distance formula forHyperX topology is as follows:

According to equation (1) average distance of a topology can obtained by

$$
\overline{\mathrm{D}}=\frac{\sum_{\mathrm{i}=1}^{\mathrm{n}} \mathrm{i} \times \mathrm{N}_{\mathrm{i}}}{\mathrm{N}-1}
$$

The unknown term of this expressionis $\mathrm{N}_{\mathrm{i}}$ that is defined by the number of terminals located in distance $i$ from the central of a specific node. For example, a group of terminals that have difference only in one dimension will be equal to

$$
\mathrm{N}_{1}=\mathrm{T} \times \sum\left(\mathrm{r}_{\mathrm{i}}-1\right), \mathrm{i} \in\{1,2, \ldots, \mathrm{n}\}
$$

Now, number of terminals that are located in a two hops distance from the central node can be calculated by computing the number of a group of terminals which differ in their dimensions and central node's dimension is two. In mathematical expression we have

$$
\mathrm{N}_{2}=\mathrm{T} \times \sum\left(\mathrm{r}_{\mathrm{i}}-1\right)\left(\mathrm{r}_{\mathrm{j}}-1\right), \quad \mathrm{i}, \mathrm{j} \in\{1,2, \ldots, \mathrm{n}\}, \mathrm{i} \neq \mathrm{j}(14)
$$

With continuation of this trend, the number of terminalslocated in distance $i$ from the central of a specific node can be expressed as follows

$$
\mathrm{N}_{\mathrm{i}}=\mathrm{T} \times \sum\left(\mathrm{r}_{\mathrm{i}}-1\right)\left(\mathrm{r}_{\mathrm{j}}-1\right)\left(\mathrm{r}_{\mathrm{i}}-1\right), \mathrm{i}, \mathrm{j}, \mathrm{k}, \ldots \in\{1,2, \ldots, \mathrm{n}\}, \mathrm{i} \neq \mathrm{j} \neq \mathrm{k} \neq \ldots
$$


We assume that number of switches in each dimensionis same as others; i.e., $1 \leq \mathrm{i} \leq \mathrm{n}, \mathrm{r}_{\mathrm{i}}=$ rThusfor $\mathrm{N}_{\mathrm{i}}$ we will havetothe following simpler form

$$
\mathrm{N}_{\mathrm{i}}=\mathrm{T}\left(\begin{array}{c}
\mathrm{n} \\
\mathrm{i}
\end{array}\right)(\mathrm{r}-1)^{\mathrm{i}}
$$

With replacing the above expression in equation (1) we have,

$$
\overline{\mathrm{D}}=\frac{\mathrm{T} \cdot \sum_{\mathrm{i}=1}^{\mathrm{n}} \mathrm{i} \cdot\left(\begin{array}{c}
\mathrm{n} \\
\mathrm{i}
\end{array}\right)(\mathrm{r}-1)^{\mathrm{i}}}{\mathrm{N}-1}
$$

Here, with simplifying, we want to achieve a proper formula foraverage distance of HyperX topology.According to the theory of combinatorics [8] we have

$$
(1+x)^{n}=\sum_{i=1}^{n}\left(\begin{array}{c}
n \\
i
\end{array}\right)(x)^{i}
$$

And with differentiation of both sides we get

$$
n(1+x)^{n-1}=\sum_{i=1}^{n} i \cdot\left(\begin{array}{c}
n \\
i
\end{array}\right)(x)^{i-1}
$$

By multiplying Eq. (19) by $x$ we obtain

$$
n x(1+x)^{n-1}=\sum_{i=1}^{n} i \cdot\left(\begin{array}{c}
n \\
i
\end{array}\right)(x)^{i}
$$

Finally, with replacing $\mathrm{x}=\mathrm{r}-1$ in the above equation we get

$$
n(r-1)(r)^{n-1}=\sum_{i=1}^{n} i \cdot\left(\begin{array}{c}
n \\
i
\end{array}\right)(r-1)^{i}
$$


So, the average distance in a regular HyperX can be calculated by

$$
\bar{D}=\frac{T \cdot n(r-1)(r)^{n-1}}{N-1}
$$

Similarly

References

$$
\overline{\mathrm{D}}=\mathrm{n} \cdot \frac{\mathrm{r}-1}{\mathrm{r}} \cdot \frac{\mathrm{N}}{\mathrm{N}-1}
$$

[1] W.J. Dally, B. Towles, Principles and practices of interconnection networks, Morgan Kaufmann Publishers, 2004.

[2] J. Kim, High-Radix Interconnection Networks, PhD Thesis, Stanford University, 2008.

[3] J. Kim, W.J. Dally, D. Abts, Flattened butterfly: a cost-efficient topology for high-radix networks, In ISCA '07: Proceedings of the 34th annual international symposium on Computer architecture, San Diego, California, USA, June (2007) 126-137.

[4] J. H. Ahn, N. Binkert, A. Davis, M. McLaren, R. S. Schreiber, HyperX: Topology, Routing, and Packaging of Efficient Large-Scale Networks, Proceedings of the Conference on High Performance Computing Networking, Storage and Analysis (2009) 1-11.

[5] L. N. Bhuyan, D. P. Agrawal, Generalized Hypercube and Hyperbus Structures for a Computer Network, IEEE Transactions on Computers, Vol.33, No. 4 (1984) $323-333$.

[6] J. Dégila, B. Sansò, A Survey of Topologies and Performance Measures for Large Scale Networks, IEEE Communications Surveys and Tutorials Magazine, Fourth Quarter, Vol. 6, No. 3(2004) 2-15.

[7] C. Leiserson, Fat-trees: Universal Networks for Hardware Efficient Supercomputing, IEEE Transactions on Computers, Vol. 34, (1985) 892-901.

[8] R. P. Grimaldi, Discrete and combinatorial mathematics: an applied introduction, 5th edn. Addison Wesley, Reading, 2004.

[9] S. Azizi, F. Safaei, N. Hashemi, On the topological properties of HyperX, The Journal of Supercomputing Vol. 66, No. 1, (2013) 572-593.

[10] E. Abuelrub, A Comparative Study on the Topological Properties of Hyper-Mesh Interconnection Network, Proceedings of World Congress on engineering, London, UK, Vol. 1, (2008) 9-5.

[11] B. Li, A. Ganz, Virtual Topologies for WDM Star LANs - the Regular Structures Approach, in INFOCOM'92. Eleventh Annual Joint Conference of the IEEE Computer and Communications Societies, (1992) 2134-2143. 